\title{
Joint Optimization Of Transceivers With Fractionally Spaced Equalizers
}

\author{
Ching-Chih Weng and P. P. Vaidyanathan \\ Dept. of Electrical Engineering, MC 136-93 \\ California Institute of Technology, Pasadena, CA 91125, USA \\ E-mail: cweng@caltech.edu, ppvnath@systems.caltech.edu
}

\begin{abstract}
In this paper we propose a method for joint optimization of transceivers with fractionally spaced equalization (FSE). We use the effective single-input multiple-output (SIMO) model for the fractionally spaced receiver. Since the FSE is used at the receiver, the optimized precoding scheme should be changed correspondingly. Simulation shows that the proposed method demonstrates remarkable improvement for jointly optimal linear transceivers as well as transceivers with decision feedback.

Index Terms - Transceiver Optimization, Fractionally Spaced Equalizers, SIMO Models, Decision Feedback, BER Minimization.
\end{abstract}

\section{INTRODUCTION}

In recent years, a large amount of work has been done on the joint design of transmitter and receiver for communications over multiple-input multiple-output channels [2], [5], [9], [12], [15]. The problem is important because it arises in many different scenarios, such as discrete multitone systems used in DSL (digital subscriber line) technology, orthogonal frequency division multiplexing (OFDM) systems, or when multiple antennas are used at both sides of a wireless link.

Based on the assumption of having perfect channel state information, the transmitter can use appropriate precoding, and jointly with the equalization scheme at the receiver side, better performance can be achieved. It is known that the optimal equalization technique is the computationally complex maximum likelihood receiver. However, due to the heavy computation load, usually the linear precoding and equalization scheme, or other suboptimal techniques, such as linear precoder with decision feedback equalizers, are utilized. Under those schemes, several different performance criteria have been used to measure the quality of communications, and optimization problems have been considered by a number of authors [9], [12].

It has been proved that when the fractionally spaced equalizer is used, the system will be more robust to the choice of timing phase [14]. This is especially advantageous if linear equalization is used [11]. Also, zero forcing (ZF) equalizers are often preferred for their simplicity. With systems having minimum redundancy, equalizers can in principle amplify channel noise in an unbounded manner (e.g., a cyclic prefix system used with a channel having a zero at a DFT frequency). The use of oversampling solves these problems because direct inversion of channel DFT is then avoided [8].

In this work, we propose to use the fractionally spaced equalizer at the receiver together with appropriate precoding. Since a fractionally spaced equalization will be used at the receiver, the optimization of the precoder should be changed accordingly. This scheme will yield better performance compared to SSE, as we shall see.

${ }^{1}$ This work is supported in parts by the NSF grant CCF-0428326, and the TMS scholarship 94-2-A-018 of the National Science Council of Republic of China, Taiwan.
Fractional sampling is known to convert the single-input single output (SISO) channel into single-input multi-output (SIMO) channels. This fact is exploited in blind channel equalization [3], OFDM [13], and to perform receiver timing and carrier synchronization. Since the channels and the noise samples produced by fractional sampling in SIMO model will be correlated, the diversity will be limited [13]. The gain from fractional sampling is what we want to quantify. It depends on the continuous channel waveform, pulse shaping filter, and receiving filter. We will also give the necessary and sufficient conditions that the fractionally spaced equalizer has no gain at all.

In this paper, we extend the transceiver design technique to fractionally sampled communication systems. The paper is organized as follows. In Section II, we describe the communication system model. In Section III, we develop a method that extends the original optimal transceiver design technique to the fractional sampling case. Some deeper discussions on quantifying the benefits of oversampling are given in section IV. Section V shows simulation results for the developed theory. Section VI concludes the paper.

\section{System Model}

Consider the continuous time baseband model for a communication system as in Fig. 1. Here $p(t)$ is the transmitting filter which is assumed to be real; $c(t)$ is the continuous time baseband channel; $n(t)$ is the additive Gaussian channel noise.

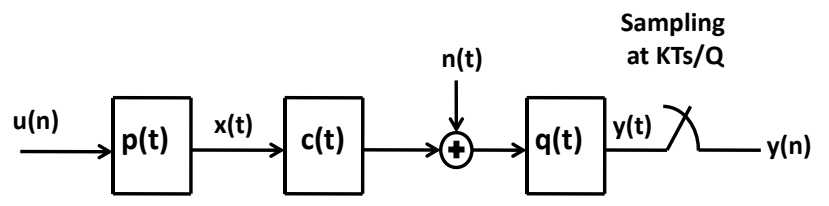

Fig. 1. Continuous time baseband model.

The transmitted continuous time signal in the baseband form is given by $x(t)=\sum_{n=0}^{P-1} u(n) p\left(t-n T_{s}\right)$, where $1 / T_{s}$ is the baud rate. The received signal prior to the sampling process is

$$
y(t)=\sum_{n=0}^{P-1} u(n) h\left(t-n T_{s}\right)+v(t) .
$$

where $h(t)$ is the impulse response of the composite channel, i.e. $h(t)=p(t) * c(t) * q(t)$. Also, $v(t)$ is the filtered channel noise, i.e. $v(t)=n(t) * q(t)$.

In the receiver end, if sampling at the baud rate, the optimal receiving filter $q(t)$ will be the one that matches the convolution of transmitting filter and the channel. In practice, this is not easy to do [10] due to hardware limitation and the unknown channel characteristics before channel estimation. 
Therefore, what is usually done is to use the filter matched to the transmitting filter, that is, $q(t)=p(-t)$. A more general front end analog filter for the blind channel identification application is discussed in [1]. It is well known that if $n(t)$ is white and the autocorrelation of the receiving filter $q(t) * q(-t)$ has the Nyquist $\left(T_{s}\right)$ property [10], $T_{s}$-spaced samples of $v(t)$ will be uncorrelated. In this paper we consider the situation that $p(t)$ is symmetric, i.e. $p(t)=p(-t)$, and with finite support. Also we assume the receiving filter matches the transmitting filter, i.e. $q(t)=p(-t)$, and that $q(t) * q(-t)$ has the Nyquist $\left(T_{s}\right)$ property. If we further assume that the channel has finite duration, the equalization can be done using just a finite number of received symbols.

Under the assumption that the channel is of finite duration, it is well known that by blocking the transmitted symbols and with suitable precoding, we can totally eliminate block-byblock interference, and equalization for inter-symbol interference can be done within each block [7]. Suppose now we assume the channel is of finite length $L \times T_{s}$ seconds, and by sampling at the baud rate, we have the equivalent block-based communication model

$$
\mathbf{Y}=\mathbf{H S}+\mathbf{V}
$$

$\mathbf{Y}$ is the vector consisting of the samples of the received signal; $\mathbf{S}$ is the transmitted vector of symbols; $\mathbf{V}$ is the additive Gaussian noise vector, and $\mathbf{H}$ is the effective channel matrix. Examples of such systems are DMT and OFDM systems [8].

The system model discussed above leads to an important transceiver design problem. This problem is extensively discussed in the literature, mainly in two categories: linear transceiver design [9] and simple nonlinear transceiver design (decision feedback equalizers with precoders) [12]. With a fractionally spaced equalizer used at the receiver, the precoder should be re-designed accordingly. This will be the main topic of this paper.

In Fig. 1 if we over-sample the received signal $y(t)$ by some integer $Q$, then its polyphase components can be expressed as (e.g. [3] and [13])

$$
y_{q}(n)=\sum_{i=0}^{P-1} u(i) h_{q}(n-i)+v_{q}(n), q=0,1, \ldots Q-1 .
$$

where $y_{q}(n)=y\left(n T_{s}+q T_{s} / Q\right), h_{q}(n)=h\left(n T_{s}+q T_{s} / Q\right)$, and $v_{q}(n)=v\left(n T_{s}+q T_{s} / Q\right)$. If we write those equations in the matrix form according to the previous discussions, we will have

$$
\mathbf{Y}_{q}=\mathbf{H}_{q} \mathbf{S}+\mathbf{V}_{q}, q=0,1, \ldots Q-1 .
$$

From the above discussions, we can see that fractional sampling in the communication system results in an SIMO model. We can group the $Q$ equations in (3) as

$$
\mathbf{Y}_{a}=\mathbf{H}_{a} \mathbf{S}+\mathbf{V}_{a} \text {. }
$$

where $\mathbf{Y}_{a}=\left[\begin{array}{llll}\mathbf{Y}_{0}^{\dagger} & \mathbf{Y}_{1}^{\dagger} & \ldots & \mathbf{Y}_{Q-1}^{\dagger}\end{array}\right]^{\dagger}, \quad \mathbf{H}_{a}=$ $\left[\begin{array}{lllll}\mathbf{H}_{0}^{\dagger} & \mathbf{H}_{1}^{\dagger} & \cdots & \mathbf{H}_{Q-1}^{\dagger}\end{array}\right]^{\dagger}$, and $\mathbf{V}_{a}=\left[\begin{array}{llll}\mathbf{V}_{0}^{\dagger} & \mathbf{V}_{1}^{\dagger} & \cdots & \mathbf{V}_{Q-1}^{\dagger}\end{array}\right]^{\dagger}$. It should be noted that, although we have multiple receptions of transmitted signal due to fractional sampling, those multiple receptions are correlated. For example, the entries in the matrix $\mathbf{H}_{q}$ are related for different $q$ 's. This correlation is related to the transmitting filter, the channel, and the receiving filter. Since $n(t)$ is white and $q(t) * q(-t)$ is Nyquist $\left(T_{s}\right)$, the covariance matrix of $\mathbf{V}_{q}$ is diagonal. However, the cross-covariance between $\mathbf{V}_{q_{1}}$ and $\mathbf{V}_{q_{2}}$ will not be zero in general for different $q_{1}$ and $q_{2}$. In order to fully address the effect of fractional sampling in the transceiver design problem, we should consider the multiple reception model as the function of the transmitting filter and the receiving filter as well [13].

Now, it is well known that the correlation function of $v(t)$ in (1) is $\sigma_{v}^{2} P_{c}(t)$, where $P_{c}(t)$ is the autocorrelation function of $p(t)$, and $\sigma_{v}^{2}$ is the noise power. Let us compute the covariance matrix of the noise vector $\mathbf{V}_{a}$.

$$
\begin{aligned}
E\left[v_{q_{1}}\left(k_{1}\right) v_{q_{2}}^{*}\left(k_{2}\right)\right] & =E\left[v\left(k_{1} T_{s}+\frac{q_{1} T_{s}}{Q}\right) v^{*}\left(k_{2} T_{s}+\frac{q_{2} T_{s}}{Q}\right)\right] \\
& =\sigma_{v}^{2} P_{c}\left(\left(k_{2}-k_{1}\right) T_{s}+\left(q_{2}-q_{1}\right) T_{s} / Q\right) .
\end{aligned}
$$

The covariance matrix of $\mathbf{V}_{a}$ can be computed from $P_{c}(t)$. For example, suppose $P_{c}(t)$ is of finite length $T_{s}$, and we define $\alpha:=P_{c}(0) / P_{c}\left(T_{s} / 2\right)$. For the case when $Q=2$, the noise covariance matrix will be

$$
E\left[\mathbf{V}_{a} \mathbf{V}_{a}^{\dagger}\right]=\sigma_{v}^{2}\left(\begin{array}{cc}
\mathbf{I} & \mathbf{A}^{\dagger} \\
\mathbf{A} & \mathbf{I}
\end{array}\right)
$$

where $\sigma_{v}^{2}$ is the noise variance, and $\mathbf{A}$ is a Toeplitz matrix with the first row $[\alpha, \alpha, 0, \cdots, 0]$, and the first column $[\alpha, 0,0, \cdots, 0]^{\prime}$. When $Q$ equals some other integer, a similar approach can be used to find $E\left[\mathbf{V}_{a} \mathbf{V}_{a}^{\dagger}\right]$.

However, the FSE transceiver design problem can always be rearranged such that the covariance matrix is identity, as we shall do at the beginning of Section III.

\section{Optimization OF TRANSCEIVER DESIGN UNDER FRACTIONALLY SPACED EQUALIZATION}

The tranceiver design problem can be explained using Fig. 2(a) and Fig. 2(b), for linear transceivers, and DFE with precoders, respectively. We assume $\mathbf{s}$ and $\mathbf{q}$ are zero mean and

$$
E\left(\begin{array}{c}
\mathbf{s} \\
\mathbf{q}
\end{array}\right)\left(\begin{array}{l}
\mathbf{s} \\
\mathbf{q}
\end{array}\right)^{\dagger}=\left(\begin{array}{cc}
\sigma_{s}^{2} \mathbf{I}_{\mathbf{M}} & \mathbf{0} \\
\mathbf{0} & \sigma_{q}^{2} \mathbf{I}_{\mathbf{J}}
\end{array}\right)
$$

The transceiver design problem can be viewed as designing the precoder matrix $\mathbf{F}$, and the equalizer matrix $\mathbf{G}$, (also the feedback filter $\mathbf{B}$ if DFE is used) according to the given channel $\mathbf{H}$ with additive noise $\mathbf{q}$, subject to some performance criteria.

In general, the noise covariance matrix $\mathbf{R}_{q q}$ is not an identity matrix. Since $\mathbf{R}_{q q}$ is Hermitian and positive definite, we can write the Cholesky decomposition of it as $\mathbf{R}_{q q}=\mathbf{R} \mathbf{R}^{\dagger}$, for some appropriate $\mathbf{R}$. Now we define $\mathbf{q}_{1}=\mathbf{R}^{-1} \mathbf{q}$ and consider the middle part of in Fig. 2(a) and Fig. 2(b) as in Fig. 2(c). The effective noise $\mathbf{q}_{1}$ has covariance matrix equal to identity, and we can design the corresponding precoder $\mathbf{F}$ and the equalizer $\mathbf{G}_{1}$ according to the effective channel $\mathbf{H}_{1}$, where $\mathbf{G}_{1}=\mathbf{G R}$ and $\mathbf{H}_{1}=\mathbf{R}^{-1} \mathbf{H}$, as in Fig. 2(d). This says that with no loss of generality, we can design the transceiver assuming additive white Gaussian noise samples.

The joint optimization of the systems in Fig. 2(a) [9] and Fig. 2(b) [12] has been discussed by several authors. We will show how to apply the transceiver design technique with a fractionally spaced equalizer used at the receiver.

If we over-sample the received waveform $y(t)$ by 2 times the symbol rate, as in (4), the received signal has the form

$$
\mathbf{Y}_{a}=\left(\begin{array}{c}
\mathbf{Y}_{0} \\
\mathbf{Y}_{1}
\end{array}\right)=\left(\begin{array}{l}
\mathbf{H}_{0} \\
\mathbf{H}_{1}
\end{array}\right) \mathbf{S}+\left(\begin{array}{l}
\mathbf{V}_{0} \\
\mathbf{V}_{1}
\end{array}\right)
$$

Suppose that the baseband channel noise, $n(t)$ in Fig. 1 is white, so the covariance matrix of $\mathbf{V}_{0}$ is identity times some constant. However, the covariance matrix of $\mathbf{V}_{a}$ will not be 

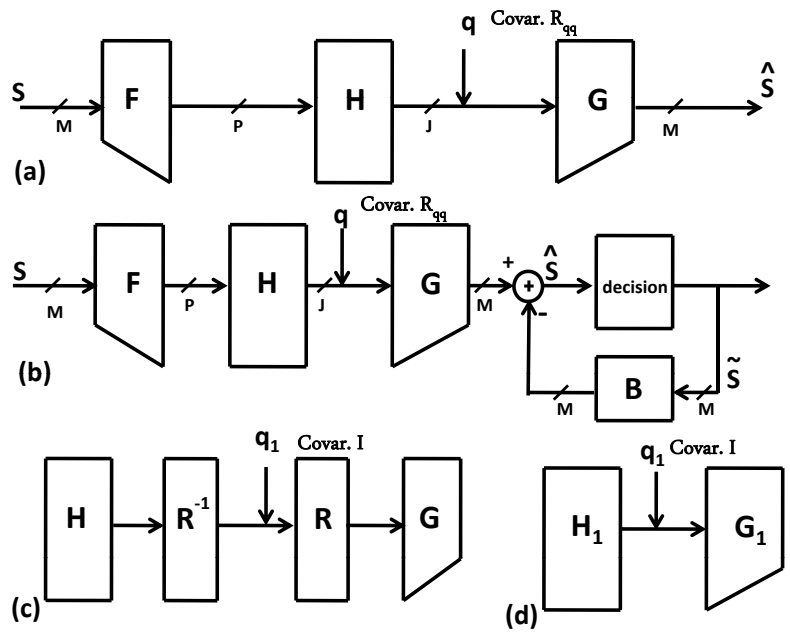

Fig. 2. (a) The linear transceiver system with noise covariance $\mathbf{R}_{q q}$. (b) The DFE system with linear precoder, with noise covariance $\mathbf{R}_{q q}$. (c) The effective additive-white noise system. (d) Absorbing $\mathbf{R}^{-1}$ and $\mathbf{R}$ into the the channel and the receiver.

an identity matrix, but will be as in (5). We can write the Cholesky factorization of the normalized covariance matrix of $\mathbf{V}_{a}$ as

$$
\frac{1}{\sigma_{v}^{2}} E\left[\mathbf{V}_{a} \mathbf{V}_{a}^{\dagger}\right]=\left(\begin{array}{cc}
\mathbf{I} & \mathbf{A}^{\dagger} \\
\mathbf{A} & \mathbf{I}
\end{array}\right)=\mathbf{L L}^{\dagger}
$$

where we choose $\mathbf{L}$ to be lower triangular. Because the upperleft part of $\frac{1}{\sigma_{v}^{2}} E\left[\mathbf{V}_{a} \mathbf{V}_{a}^{*}\right]$ is an identity matrix, it is trivial to see that

$$
\mathbf{L}=\left(\begin{array}{cc}
\mathbf{I} & \mathbf{0} \\
\mathbf{L}_{21} & \mathbf{L}_{22}
\end{array}\right)
$$

and

$$
\mathbf{L}^{-1}=\left(\begin{array}{cc}
\mathbf{I} & \mathbf{0} \\
\mathbf{B} & \mathbf{D}
\end{array}\right)
$$

for appropriate $\mathbf{L}_{21}, \mathbf{L}_{22}, \mathbf{B}$, and $\mathbf{D}$. Since

$$
\mathbf{L L}^{\dagger}=\left(\begin{array}{cc}
\mathbf{I} & \mathbf{L}_{21}^{\dagger} \\
\mathbf{L}_{21} & \mathbf{L}_{21} \mathbf{L}_{21}^{\dagger}+\mathbf{L}_{22} \mathbf{L}_{22}^{\dagger}
\end{array}\right)
$$

we have $\mathbf{L}_{21}=\mathbf{A}$. By $\mathbf{L L}^{-1}=\mathbf{I}$, we have $\mathbf{D}=\mathbf{L}_{22}^{-1}$, and $\mathbf{B}=-\mathbf{L}_{22}^{-1} \mathbf{L}_{21}$. Thus we define the whitened effective channel with the transfer matrix

$$
\mathbf{H}_{e f f}:=\mathbf{L}^{-1} \mathbf{H}_{a}=\left(\begin{array}{c}
\mathbf{H}_{0} \\
-\mathbf{L}_{22}^{-1}\left(\mathbf{H}_{1}-\mathbf{L}_{21} \mathbf{H}_{0}\right)
\end{array}\right)
$$

By multiplying $\mathbf{L}^{-1}$ on both side of (4), we have

$$
\mathbf{L}^{-1} \mathbf{Y}_{a}=\mathbf{H}_{e f f} \mathbf{S}+\mathbf{L}^{-1} \mathbf{V}_{a}
$$

where the covariance matrix of $\mathbf{L}^{-1} \mathbf{V}_{a}$ is an identity matrix times a constant. Therefore we can design the transceiver of the fractionally spaced system as in (10), with effective channel $\mathbf{H}_{e f f}$, while the effective noise $\mathbf{L}^{-1} \mathbf{V}_{a}$ is white.

It is intuitive that the fractionally spaced system will perform better than the symbol spaced one. We will give detail discussion in the following section.

\section{ANALYSIS AND COMPARISON}

In this section we will discuss how the gain comes from using fractional sampling technique with precoders.

The average mean-squared error $\phi$ in an MMSE transceiver, with or without the zero forcing constraint, and with or without decision feedback, can be shown [12], [9] to be a function of the $M$ dominant singular values of the channel $\mathbf{H}$ :

$$
\phi=f\left(\sigma_{1}, \sigma_{2}, \ldots \sigma_{M}\right)
$$

where $\sigma_{1}, \sigma_{2}, \cdots, \sigma_{M}$ denote the first $M$ dominant singular values of the channel matrix $\mathbf{H}$. Similarly the minimized power in the case of optimal transceivers with bit allocation and fixed QoS constraints also has this form [7]. Furthermore, in all of the above cases $f(\cdot)$ is a decreasing function of each argument $\sigma_{k}$. Based only on this observation we now show that the use of oversampling improves the performance except in some rare situations which we shall point out. The results are based on Weyl's theorem [4]:

Lemma 1. (p.181 in [4]): Let $\mathbf{A}, \mathbf{B} \in \mathbf{M}_{n}$ be Hermitian and let the eigenvalues $\lambda_{i}(\mathbf{A}), \lambda_{i}(\mathbf{B})$, and $\lambda_{i}(\mathbf{A}+\mathbf{B})$ be arranged in decreasing order. Then for each $k=1,2, \ldots \ldots, n$ we have

$$
\lambda_{k}(\mathbf{A})+\lambda_{n}(\mathbf{B}) \leq \lambda_{k}(\mathbf{A}+\mathbf{B}) \leq \lambda_{k}(\mathbf{A})+\lambda_{1}(\mathbf{B})
$$

Using lemma 1, we can prove the following theorem:

Theorem 1. The communication system in Fig. 1 with optimal transceiver matrices, will have smaller MMSE for larger $Q$.

Proof: First let $Q=2$, then we define $\mathbf{H}_{1}^{\prime}:=$ $-\mathbf{L}_{22}^{-1}\left(\mathbf{H}_{1}-\mathbf{L}_{21} \mathbf{H}_{0}\right)$. Here the matrices $\mathbf{H}_{0}, \mathbf{H}_{1}$, and $\mathbf{H}_{1}^{\prime}$ are all with dimension $J \times P$. The singular values of $\mathbf{H}_{\text {eff }}$ are the eigenvalues of $\mathbf{H}_{0} \mathbf{H}_{0}^{\dagger}+\mathbf{H}_{1}^{\prime} \mathbf{H}_{1}^{\prime}$. Since $\mathbf{H}_{0} \mathbf{H}_{0}^{\dagger}$ and $\mathbf{H}_{1}^{\prime} \mathbf{H}_{1}^{\prime \dagger}$ are both Hermitian and positive semi-definite matrices, all of their eigenvalues are nonnegative. By applying Lemma 1,

$\lambda_{k}\left(\mathbf{H}_{e f f} \mathbf{H}_{e f f}^{\dagger}\right) \geq \lambda_{k}\left(\mathbf{H}_{0} \mathbf{H}_{0}^{\dagger}\right)+\lambda_{J}\left(\mathbf{H}_{1}^{\prime} \mathbf{H}_{1}^{\prime \dagger}\right) \geq \lambda_{k}\left(\mathbf{H}_{0} \mathbf{H}_{0}^{\dagger}\right)$

where the first inequality is from Lemma 1, and the second inequality is from the positive semi-definiteness of $\mathbf{H}_{1}^{\prime} \mathbf{H}_{1}^{\prime}$.

Since the singular values of the effective channel will be greater, the average mean-squared error $\phi$ will become smaller due to fractional sampling. The proof for $Q>2$ is similar.

Oversampling offers no gain if and only if $\lambda_{k}\left(\mathbf{H}_{\text {eff }} \mathbf{H}_{\text {eff }}^{\dagger}\right)=\lambda_{k}\left(\mathbf{H}_{0} \mathbf{H}_{0}^{\dagger}\right)$ for all $k$, which in turn is true if and only if $\mathbf{H}_{1}^{\prime}=\mathbf{0}$. From the definition of $\mathbf{H}_{1}^{\prime}$ we see that this is equivalent to $\mathbf{H}_{1}=\mathbf{L}_{21} \mathbf{H}_{0}$. Another way to interpret this is as follows: the components of $\mathbf{V}_{a}$ are in general correlated, and the pre-multiplication with $\mathbf{L}^{-1}$ in (10) makes the covariance of $\mathbf{L}^{-1} \mathbf{V}_{a}$ identity. Under this "uncorrelated frame of reference," the lower half of $\mathbf{H}_{\text {eff }} \mathbf{S}$ in Eq. (10) is a measure of the additional information gained due to oversampling. This "innovations component" is zero if and only if the condition $\mathbf{H}_{1}^{\prime}=\mathbf{0}$ arises.

Consider an example. Let $c(t)=\sum_{i=0}^{L-1} \alpha_{i} \delta\left(t-i T_{s}\right)$, and assume $q(t)=p(-t)$ as before. In this case the effective channel is $h(t)=\sum_{i=0}^{L-1} \alpha_{i} P_{c}\left(t-i T_{s}\right)$, where $P_{c}(\tau)$ is the autocorrelation of $p(t)$. But the noise correlation at the output of $q(t)$ is also $P_{c}(\tau)$. If we assume there is no timing error due to sampling at the receiver, it can be shown in this case that $\mathbf{H}_{1}^{\prime}=\mathbf{0}$, and there is nothing to be gained by oversampling by two; in fact this is the case regardless of what the oversampling ratio is! 


\section{Numerical Results}

In this section we present our simulation results relevant to the discussion carried out in previous sections. Consider a channel of the form $c(t)=\sum_{i=0}^{m-1} \alpha_{i} \delta\left(t-\tau_{i}\right)$, where $\alpha_{i}$ are random complex Gaussian variables and $\delta(t)$ is the Dirac delta function. Here $m=20$, and $\tau_{i}$ are randomly chosen in the range of $0 \leq \tau_{i} \leq 12 T_{s}$. This is the model which is usually adopted in wireless communication systems, representing the channel that contains discrete multi-path components [10]. Assume the transmitting pulse $p(t)$ is a truncated Gaussian with support in $\left[-T_{s} / 2, T_{s} / 2\right]$, and the receiver pulse $q(t)=$ $p(-t)=p(t)$. The effective channel delay spread is assume to be within $12 T_{s}$, so that the equivalent discrete time FIR channel has order $L=12$. Using a zero-padding size $L=12$ we obtain freedom from inter-block interference. With block size $M=64$ we have, in the notation of Fig. 2(a), $M=P=$ 64 , and $J=64+12=76$. Each symbol is from a QPSK constellation.

For the linear MMSE transceiver designed using standard techniques [9], the simulated SER performance plots are shown in Fig. 3 for various oversampling factors. The plots show that when we move from the SSE to FSE with oversampling ratio of two, there is a large improvement in performance. As the oversampling ratio is increased further the performance continues to improve, though by smaller amounts.

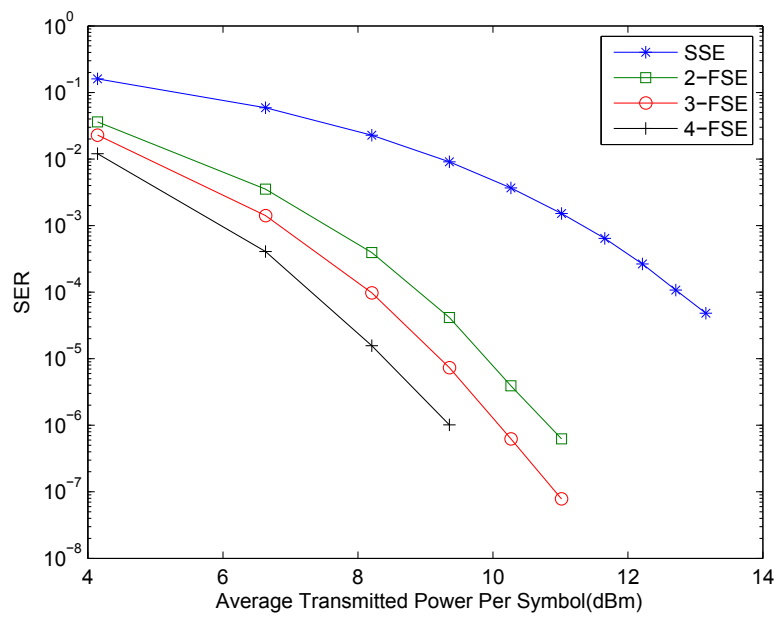

Fig. 3. Performance of fractional sampling system with different sampling rates. Noise power is $3.5 \mathrm{dBm}$ per received symbol.

Fig. 4 compares the simulated SER plots for three optimal systems with and without FSE (with factor of two oversampling). The first system is the MMSE system as in Fig. 3 . The second system is the MMSE system with precoder constrained to be unitary apriori. The third system is the optimal transceiver which uses decision feedback [12]. In all cases it is clear that oversampling results in significant improvement.

It is assumed in all plots that the MMSE systems are also minimum SER systems. This can be ensured for linear transceivers by inserting a Hadamard matrix at the transmitter and its inverse at the receiver [9], [6], [2] (for DFE transceivers the method is more involved; see [12]).

\section{CONCLUding REMARKS}

We showed that the performance of jointly optimal transceivers can further be improved by using oversampled

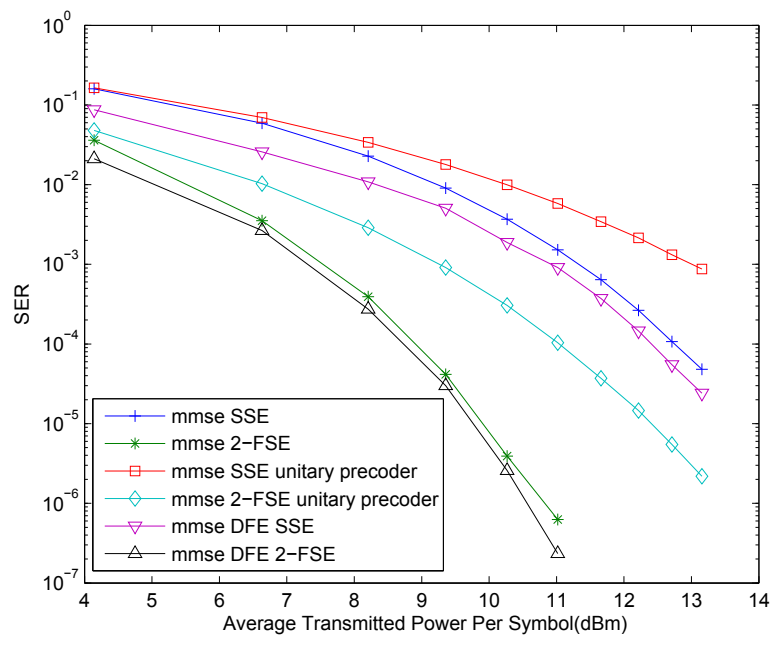

Fig. 4. Performance of SSE and FSE system, with MMSE transceiver. Noise power is $3.5 \mathrm{dBm}$ per received symbol.

receivers. Analysis of the conditions under which no improvement is possible shows that such a situation arises only if the noise covariance and effective channel in the oversampled system satisfy a certain equality. As this is unlikely to happen in practice, improved performance can always be expected. The simulations presented here demonstrate remarkable improvement in performance for jointly optimal linear transceivers as well as transceivers with decision feedback.

\section{REFERENCES}

[1] D. J. Borah, R. A. Kennedy, Z. Ding, and I. Fijalkow, "Sampling and prefiltering effects on blind equalizer design," IEEE Trans. Sig. Proc. pp. 209 - 218, Jan. 2001

[2] Y. Ding, T. N. Davidson, Z. Luo, and K. M. Wong, "Minimum BER precoders for zero-forcing equalization," IEEE Trans. Sig. Proc. pp. 2410 -2423 , Sep. 2003.

[3] G. Giannakis, C. Tepedelenlioglu, "Basis expansion models and diversity techniques for blind identification and equalization of time-varying channels," Proc. of the IEEE, pp. 1969 - 1986, Oct. 1998.

[4] R. A. Horn, C. R. Johnson, "Matrix analysis," Cambridge Univ. Press 1985 .

[5] Y. Jiang, J. Li, and W. W. Hager "Joint transceiver design for MIMO communications using geometric mean decomposition, "IEEE Trans. Sig. Proc., pp.3791-3803, Oct. 2005

[6] Y. P. Lin, and S. M. Phoong, "BER minimized OFDM systems with channel independent precoders," IEEE Trans. Sig. Proc. pp. 2369 - 2380, Sep. 2003.

[7] Y. P. Lin, and S. M. Phoong, "Optimal ISI-Free DMT transceivers for distorted channels with colored noise," IEEE Trans. Sig. Proc. pp. 2702 -2712 , Nov. 2001.

[8] B. Vrcelj, and P. P. Vaidyanathan "Fractional biorthogonal partners in channel equalization and signal interpolation," IEEE Trans. Sig. Proc. pp. 1928 - 1940, Jul. 2003.

[9] D. P. Palomar, J. M. Cioffi, and M. A. Lagunas, "Joint Tx-Rx beamforming design for multicarrier MIMO channels: a unified framework for convex optimization," IEEE Trans. Sig. Proc. pp. 2381 - 2401, Sept 2003.

[10] G. Proakis, "Digital communication," 4th Ed. New York: McGraw-Hill, 2001.

[11] J. Salz, "On mean-square decision feedback equalization and timing phase," IEEE Trans. comm. pp. 1471 - 1476, Dec. 1977.

[12] M. B. Shenouda, T. N. Davidson, "A framework for designing MIMO systems with decision feedback equalization or Tomlinson-Harashima precoding," Proc. of the ICASSP, pp. 209-212, April. 2007.

[13] C. Tepedelenlioglu, R. Challagulla, "Low-complexity multipath diversity through fractional sampling in OFDM," IEEE Trans. Sig. Proc., pp. $3104-$ 3116, Nov. 2004

[14] G. Ungerboeck, "Fractional tap-spacing equalizers and consequences for clock recovery in data modems," IEEE Trans. comm. pp. 856 - 864 , Aug. 1976.

[15] F. Xu, T. N. Davidson, J. K. Zhang, S. S. Chan, and K. M. Wong "Design of block transcivers with MMSE decision feedback detection," Proc. of the ICASSP, pp. 1109 - 1112, March. 2004. 